\title{
MORTALIDAD POR BACTERIEMIA CAUSADA POR Escherichia coli Y Klebsiella spp. PRODUCTORAS DE BETA LACTAMASAS DE ESPECTRO EXTENDIDO: COHORTE RETROSPECTIVA EN UN HOSPITAL DE LIMA, PERÚ
}

\author{
Diego Adrianzén ${ }^{1,2, a}$, Ángela Arbizu ${ }^{1, a}$, Jimmy Ortiz ${ }^{1, a}$, Frine Samalvides ${ }^{1,3, b}$
}

\section{RESUMEN}

Objetivos. Evaluar los factores asociados a la mortalidad causada por bacteriemias por Escherichia coli y Klebsiella spp. productoras de beta lactamasas de espectro extendido (BLEE). Materiales y Métodos. Se realizó un estudio de cohortes retrospectivo, que incluyó 85 pacientes mayores de 16 años con diagnóstico de bacteriemia por Escherichia coli o Klebsiella spp. hospitalizados entre 2006 y 2008 en el Hospital Nacional Cayetano Heredia. Las cohortes se clasificaron de acuerdo a la producción de BLEE según los resultados de los hemocultivos. Se evaluaron los factores asociados a la mortalidad cruda y atribuible empleando regresión de Poisson en un modelo multivariado, con lo que se obtuvo riesgos relativos ajustados (RRa). Además, se construyeron curvas de mortalidad. Resultados. Se encontró que el $35,3 \%$ de las bacteriemias fueron debidas a cepas productoras de BLEE. El análisis de la mortalidad cruda mostró una mayor mortalidad en el grupo de cepas productoras de BLEE (63,3\%). EI RRa fue de 1,5 (IC95\%: $1,02-2,3)$. En el caso de mortalidad atribuible, la proporción también fue mayor (63,3\%), el RRa fue de 1,9 (IC95\%: $1,2-2,9)$. El uso de catéter venoso central fue otro factor asociado tanto a la mortalidad cruda (RRa= 2,4; IC95\%: 1,2$4,8)$ como a la mortalidad atribuible (RRa= 3,8; IC95\%: 1,6-8,8). Conclusiones. La producción de BLEE es un factor de riesgo independiente para mortalidad por bacteriemia causada por E. coli y Klebsiella spp. Su presencia debe evaluarse tras la sospecha diagnóstica y la elaboración de la terapéutica inicial, lo que podría disminuir la mortalidad por esta causa.

Palabras clave: betalactamas; Escherichia coli; Klebsiella; Mortalidad; Bacteremia (fuente: DeCS BIREME).

\section{MORTALITY CAUSED BY BACTEREMIA Escherichia coli AND Klebsiella spp. EXTENDED-SPECTRUM BETA-LACTAMASE-PRODUCERS: A RETROSPECTIVE COHORT FROM A HOSPITAL IN LIMA, PERU}

\begin{abstract}
Objectives. To evaluate the factors associated to mortality caused by bacteremia due to Escherichia coli and Klebsiella spp. producers of extended-spectrum beta-lactamase (ESBL). Materials and methods. We performed a retrospective cohort study, including 85 patients older than 16 and diagnosed with bacteremia by Escherichia coli or Klebsiella spp., hospitalized between 2006 and 2008 in Cayetano Heredia National Hospital. Cohorts were classified according to the ESBL production following blood culture results. Factors associated to gross and attributable mortality were evaluated using the Poisson regression in a multivariate model, through which adjusted relative risks (ARRs) were obtained. Mortality curves were also built. Results. $35.3 \%$ of bacteremia cases were caused by ESBL-producing strains. The analysis of gross mortality showed a higher mortality rate in the group with ESBL producing strains $(63.3 \%)$, ARR being 1.5 (Cl 95\%: 1.02-2.3). In the case of the attributable mortality, the proportion was also higher (63.3\%), ARR being 1.9 (CI 95\%: 1.2-2.9). The use of a central venous catheter was another factor associated to both gross mortality (ARR=2.4; $\mathrm{Cl} 95 \%: 1.2-4.8)$ and attributable mortality (ARR=3.8; $\mathrm{Cl} 95 \%: 1.6-8.8)$. Conclusions. The production of ESBL is an independent risk factor for bacteremia mortality caused by E. coli and Klebsiella spp. Its presence should be evaluated following diagnosis consideration and initial therapy elaboration, which could in turn decrease the mortality by this cause.
\end{abstract}

Key words: beta-Lactams; Escherichia coli; Klebsiella; Mortality; Bacteremia (source: MeSH NLM).

Facultad de Medicina "Alberto Hurtado", Universidad Peruana Cayetano Heredia. Lima, Perú.

Unidad de Conocimiento y Evidencia, CONEVID, Universidad Peruana Cayetano Heredia. Lima, Perú.

Instituto de Medicina Tropical "Alexander von Humboldt", Universidad Peruana Cayetano Heredia. Lima, Perú.

Médico cirujano, ${ }^{\mathrm{b}}$ médico infectólogo, magister en Epidemiología Clínica

Recibido: 03-07-12 Aprobado: 06-03-13

Citar como: Adrianzén D, Arbizu A, Ortiz J, Samalvides F. Mortalidad por bacteriemia causada por Escherichia coli y Klebsiella spp. productoras de beta lactamasas de espectro extendido: cohorte retrospectiva en un hospital de Lima, Perú. Rev Peru Med Exp Salud Publica. 2013;30(1):18-25. 


\section{INTRODUCCIÓN}

Las bacteriemias son infecciones nosocomiales frecuentes, en pacientes criticos representan una grave complicación que puede afectar de manera negativa el pronóstico del paciente (1). Ellas pueden ser primarias o secundarias. Las bacteriemias primarias se presentan en ausencia de otra infeccion concomitante con el paciente, se le conoce como infección del torrente sanguíeno (InfTS); en tanto que las bacteriemias secundarias se dan en presencia de una infección severa concomitante (2), en estos casos el agente etiológico más frecuentemente implicado es la Escherichia coli (E. coli), la cual es la primera causa en bacteriemia adquirida en la comunidad, y la tercera más común en bacteriemia nosocomial ${ }^{(3)}$. Sin embargo, se ha visto un incremento a nivel mundial de las bacteriemias causadadas por otras Enterobacteriaceae, como la Klebsiella spp ${ }^{(4,5)}$. El tratamiento primordial para ambos tipos de bacteriemias esta constituido por las oximino-cefalosporinas ${ }^{(6)}$.

Se sabe, además, que las beta lactamasas de espectro extendido (BLEE) son enzimas que le confieren resistencia, a los organismos que las producen, frente a todos los antibióticos beta-lactámicos excepto a las cefamicinas y a los carbapenemos (6,7). Los organismos productores de BLEE han causado brotes de infecciones multidrogorresistentes y altas tasas de fracaso al tratamiento empírico ${ }^{(8,9)}$, especialmente en unidades de cuidados intensivos (UCI) ${ }^{(6)}$. La E. coli y la Klebsiella pneumoniae son los productores de BLEE más comunes a nivel mundial ${ }^{(4,8)}$. Llama la atención que la $E$. coli productora de BLEE, inicialmente descrita como un patógeno nosocomial, sea hoy una causa en incremento de bacteriemia adquirida en la comunidad ${ }^{(10,11)}$.

Se ha señalado que cerca del $30 \%$ de las bacteriemias son causadas por gérmenes productores de BLEE ${ }^{(6)}$. En Latinoamérica se han informado frecuencias entre $14{ }^{(12)}$ y $45 \%{ }^{(13)}$ de cepas BLEE en bacteriemias causadas por $E$. coli, valores que se encuentran por encima de lo que se ha descrito en otras regiones, ello podría deberse al uso poco racional de las cefalosporinas y a tratamientos empíricos inadecuados. Se han señalado como factores asociados a adquirir cepas productoras de BLEE: la exposición previa a antibióticos, especialmente oximino-cefalosporinas, brotes nosocomiales, procedimientos invasivos, líneas centrales, ventilación mecánica, admisión a $\mathrm{UCl}$, inmunosupresión, comorbilidades, malignidad, hospitalizaciones en el año previo y tiempos hospitalarios prolongados $(4-6,12,14-18)$.
Los estudios sobre el impacto de la producción de BLEE en los resultados clínicos presentan hallazgos heterogéneos. Algunos estudios no han encontrado diferencias en las tasas de mortalidad comparadas en pacientes con bacteriemias producidas por cepas productoras y no productoras de BLEE (12,15-17,20), en tanto que otros han encontrado mayores tasas de mortalidad en bacteriemia entre los patógenos productores de BLEE ${ }^{(18,19,21,22)}$, además, se ha informado que los pacientes con bacteriemia por E. coli y Klebsiella spp. productoras de BLEE presentan una mayor estancia y gasto hospitalario ${ }^{(7,20)}$, un mayor fracaso de tratamientos empíricos ${ }^{(13,21)}$ y un mayor requerimiento de $\mathrm{UCl}{ }^{(17)}$.

En relación a la mortalidad por bacteriemia por cepas productoras de BLEE, los factores que han mostrado asociación incluyen: retraso de antibioticoterapia apropiada; el tratamiento empírico inadecuado; la edad; el uso de oximino-cefalosporinas como tratamiento definitivo; las comorbilidades; el origen nosocomial; el mayor tiempo hospitalario; la neutropenia; el foco de bacteriemia no identificado; el foco no urinario; el shock séptico, y la admisión a la UCl $(4-6,13-17,22,23)$.

Dado que la evidencia existente es heterogénea, este estudio busca evaluar los factores asociados a la mortalidad causada por bacteriemia por E.coli y Klebsiella spp productoras de BLEE, diferenciando la mortalidad cruda de la atribuible a estas bacterias.

\section{MATERIALES Y MÉTODOS}

\section{DISEÑO Y POBLACIÓN}

Se realizó un estudio de cohortes retrospectiva, para la cual se incluyó a todos los pacientes mayores de 16 años que fueron hospitalizados en el Hospital Nacional Cayetano Heredia entre enero de 2006 y diciembre de 2008, y que cumplían criterios de diagnóstico para bacteriemia causada por E. coli o Klebsiella spp. Se clasificó a los pacientes de acuerdo a la producción de BLEE por la cepa infectante, para ello se revisó todos los hemocultivos positivos para E.coli y Klebsiella spp., que se encontraban registrados en el Servicio de Microbiología del Laboratorio Central del mencionado hospital; adicionalmente, se revisaron las historias clínicas y se recolectó la información demográfica y clínica de todos los pacientes incluidos. El seguimiento de los pacientes comprendió el tiempo entre su admisión y el alta o fallecimiento intrahospitalario. 


\section{DEFINICIÓN DE BACTEREMIAS Y BLEE}

Se consideraron organismos productores de BLEE aquellos con tamizaje laboratorial positivo y confirmación fenotípica con antibióticos seleccionados en la prueba de difusión en disco, siguiendo los estándares de la Health Protection Agency ${ }^{(24,25)}$. Se definió bacteriemia como uno o más hemocultivos positivos para E.coli o Klebsiella spp. (1) y el foco de bacteriemia se determinó mediante aislamiento del mismo patógeno en otra zona corporal ${ }^{(1)}$. La InfTS fue considerada en pacientes con uno o más hemocultivos positivos y al menos uno de los siguientes signos 0 síntomas: fiebre, escalofríos e hipotermia; pero cuyos síntomas y resultados de laboratorio no estaban asociados a infección en otra zona corporal (1). Toda bacteriemia que ocurrió pasadas las 48 primeras horas de admisión, y aquellas que ocurrieron dentro de las 48 horas en pacientes transferidos u hospitalizados de otra institución en los 30 días previos a su admisión fueron consideradas de origen nosocomial ${ }^{(1,26)}$.

\section{MORTALIDAD CRUDA Y ATRIBUIBLE}

Se consideró mortalidad directamente atribuible aquella que ocurrió en el contexto de evidencia clínica de infección activa y hemocultivo positivo ${ }^{(7,27)}$ O en los 5 días posteriores al último hemocultivo positivo ${ }^{(28)}$. Se consideró mortalidad indirectamente atribuible en quienes la causa de fallecimiento fue compromiso orgánico secundario a bacteriemia ${ }^{(7,28)}$. La mortalidad atribuible total fue la suma de las mortalidades directa e indirectamente atribuibles ${ }^{(7,29,30)}$. La mortalidad no relacionada a bacteriemia fue aquella que ocurrió después de un episodio de bacteriemia pero por causas independientes al proceso infeccioso ${ }^{(29,30)}$. La mortalidad cruda incluyó a todas las causas de muerte durante la hospitalización, sean atribuibles o no a bacteriemia ${ }^{(10,17,30)}$.

\section{ANÁLISIS ESTADÍSTICO}

Las cepas aisladas se clasificaron como productoras o no productoras de BLEE. Se regristraron, además, las siguientes entidades como presentes o ausentes: malignidad (tumor sólido o hematológico); inmunosupresión (enfermedad autoinmune, uso de esteroides, neutropenia e infección por el virus de inmunodeficiencia humana $[\mathrm{VIH}]$ ); procedimientos invasivos (catéter urinario, catéter venoso central [CVC], ventilación mecánica); comorbilidades (diabetes, hipertensión arterial, enfermedad renal crónica, insuficiencia cardiaca, cirrosis, anemia, malnutrición), y complicaciones relacionadas a infección (shock séptico, sindrome de distrés respiratorio agudo [SDRA], insuficiencia renal aguda, desequilibrio electrolítico, alteración del sensorio, e insuficiencia hepática). En el análisis estadístico se incluyó el análisis descriptivo demográfico de la población; las variables categóricas se compararon usando chi cuadrado o prueba exacta de Fisher, las variables continuas se compararon usando $T$ de student o la prueba de U-Mann Whitney.

Los factores asociados a mortalidad cruda y atribuible, fueron evaluados en un modelo de regresión de Poisson, cuyo modelo multivariado incluyó las variables que demostraron significancia estadística en el análisis bivariado. Se empleó el test Wald para examinar la significancia global del modelo de regresión multivariada y el likelihood ratio test (LRt) para la comparación con el modelo univariado. La significancia estadística fue considerada cuando el valor de $p$ fue menor a 0,05. A través de estos análisis se calculó los riesgos relativos (RR) con un intervalo de confianza del 95\% (IC95\%) para mortalidad tanto cruda como atribuible.

Para la construcción de las curvas de mortalidad, se empleó el estimador Kaplan Meier, empleando como inicio del tiempo de seguimiento el día del diagnóstico (primer hemocultivo positivo documentado) y como desenlace la muerte del paciente. Las altas fueron consideradas como censuras administrativas. Los análisis se realizaron en el programa estadistico SPSS V.12.0.

\section{ASPECTOS ÉTICOS}

El estudio contó con la aprobación del Comité de Ética en Investigación de la Universidad Peruana Cayetano Heredia; adicionalmente, la versión final del artículo fue revisado y aprobado por la Unidad de Investigación, Ciencia y Tecnología de la Facultad de Medicina Alberto Hurtado de la Universidad Peruana Cayetano Heredia. El manejo de los datos fue confidencial y al tratarse de un estudio retrospectivo, no significó ningún riesgo para los pacientes.

\section{RESULTADOS}

Se incluyó un total de 85 pacientes: 27 en el año 2006; 27 en el 2007, y 33 en el 2008, se excluyó a dos casos en los que se encontró información laboratorial y clínica discordante, con ausencia de evidencia de un proceso infeccioso durante el curso de hospitalización. La edad promedio fue de $53,1 \pm 21,3$ años (rango 16-92), el $51,8 \%(44 / 85)$ fueron varones.

Se encontró que el 35,3\% (30/85) de las cepas estudiadas fueron productoras de BLEE, por lo que 
Tabla 1. Características de los pacientes con diagnóstico de bacteriemia en el Hospital Nacional Cayetano Heredia. Lima, 2006-2008.

\begin{tabular}{|c|c|c|c|}
\hline & $\begin{array}{c}\text { Total } \\
\text { N. }{ }^{\circ}(\%)\end{array}$ & $\begin{array}{c}\text { BLEE (+) } \\
\mathrm{N} .{ }^{\circ}(\%)\end{array}$ & $\begin{array}{c}\text { BLEE (-) } \\
\mathrm{N}^{\circ}{ }^{\circ}(\%)\end{array}$ \\
\hline \multicolumn{4}{|c|}{ Patógeno infectante } \\
\hline Escherichia coli & $62(72,9)$ & $16(53,3)$ & $46(83,6)$ \\
\hline Klebsiella spp, & $23(27,1)$ & $14(46,7)$ & $9(16,4)$ \\
\hline \multicolumn{4}{|l|}{ Sexo } \\
\hline Masculino & $44(51,8)$ & $18(60)$ & $26(47,3)$ \\
\hline Femenino & $41(48,2)$ & $12(40)$ & $29(52,7)$ \\
\hline \multicolumn{4}{|c|}{ Origen de bacteriemia } \\
\hline Nosocomial & $29(34,1)$ & $14(46,7)$ & $15(27,3)$ \\
\hline Comunitario & $56(65,9)$ & $16(53,3)$ & $40(72,7)$ \\
\hline \multicolumn{4}{|c|}{ Foco de bacteriemia } \\
\hline Urinario & $33(38,8)$ & $8(26,7)$ & $25(45,5)$ \\
\hline Gastrointestinal & $11(12,9)$ & $7(23,3)$ & $4(7,3)$ \\
\hline Pulmonar & $18(21,2)$ & $8(26,7)$ & $10(18,2)$ \\
\hline Biliar & $7(8,2)$ & $1(3,3)$ & $6(10,9)$ \\
\hline Uterino & $3(3,5)$ & $0(0)$ & $3(5,4)$ \\
\hline Otros & $5(5,9)$ & $2(6,7)$ & $3(5,4)$ \\
\hline InfTS & $8(9,4)$ & $4(13,3)$ & $4(7,3)$ \\
\hline \multicolumn{4}{|l|}{ Ingreso a UCI } \\
\hline Con ingreso & $17(20,0)$ & $8(26,7)$ & $9(16,4)$ \\
\hline Sin ingreso & $68(80,0)$ & $22(73,3)$ & $46(83,6)$ \\
\hline
\end{tabular}

la proporción entre los grupos productores y no productores de BLEE fue de 1/1,83. La proporcion anual de cepas BLEE fue de 25,$9 ; 30,8$ y $46,9 \%$ para cada año de estudio respectivamente. Otras caracteristicas de la muestra se pueden apreciar en la Tabla 1.

No se encontró diferencias con el sexo $(p=0,23)$, el origen nosocomial de la bacteriemia $(p=0,073)$, o el ingreso a la $\mathrm{UCl}(p=0,256)$. Tampoco hubo diferencias entre los grupos productores y no productores de BLEE en relación a las variables edad (medias de $52 \pm 21,4$ y $53,7 \pm 21,5$ años respectivamente $[p=0,728]$ ); tiempo total de hospitalización (medias de $24,5 \pm 30,9$ y 18,3 \pm 26,5 días $[p=0,357]$ ), el tiempo de estancia hospitalaria en $\mathrm{UCl}$ (medias de $1,9 \pm 3.9$ y $1,4 \pm 3,8$ días $[p=0,554]$ ) y el tiempo entre diagnóstico de bacteriemia, y el fallecimiento (medias de $3,1 \pm 3,9$ y $8,2 \pm 16,9$ días $[p=0,207]$ ).

Respecto a las comorbilidades, el desequilibrio electrolítico estuvo asociado a una mayor frecuencia de BLEE $(p=0,005)$. La exposición a dispositivos invasivos, presencia de inmunodeficiencia, comorbilidades y complicaciones fueron más frecuentes en el grupo BLEE; sin embargo, estas diferencias no fueron significativas (Tabla 2).
Tabla 2. Comorbilidad asociada a pacientes con bacteriemia por cepas productoras de BLEE.

\begin{tabular}{|c|c|c|c|c|}
\hline & $\frac{\text { Total }}{\mathrm{N} . .^{\circ}(\%)}$ & $\frac{\text { BLEE (+) }}{N^{\circ} .^{\circ}(\%)}$ & $\frac{\text { BLEE (-) }}{N^{\circ}(\%)}$ & $P^{*}$ \\
\hline \multicolumn{5}{|l|}{ Inmunodeficiencia } \\
\hline Autoinmune & $4(4,7)$ & $2(6,6)$ & $2(3,6)$ & $0,53^{\dagger}$ \\
\hline Corticoterapia & $4(4,7)$ & $2(6,6)$ & $2(3,6)$ & $0,53^{\dagger}$ \\
\hline VIH & $11(14,1)$ & $5(16,7)$ & $4(7,3)$ & $0,27^{\dagger}$ \\
\hline Neutropenia & $6(7,1)$ & $4(13,3)$ & $2(3,6)$ & $0,20^{\dagger}$ \\
\hline \multicolumn{5}{|c|}{ Exposición a dispositivos invasivos } \\
\hline Catéter vesical & $51(60)$ & $21(70)$ & $30(54,5)$ & 0,17 \\
\hline CVC & $32(37,6)$ & $14(46,7)$ & $18(32,7)$ & 0,21 \\
\hline Ventilación mecánica & $13(15,3)$ & $6(20)$ & $7(12,7)$ & 0,37 \\
\hline Traqueotomía & $1(1,2)$ & $0(0)$ & $1(1,8)$ & - \\
\hline \multicolumn{5}{|l|}{ Comorbilidades } \\
\hline Diabetes mellitus & $13(15,3)$ & $2(6,6)$ & $11(20)$ & $0,10^{\dagger}$ \\
\hline Hipertensión & $10(11,8)$ & $5(16,7)$ & $5(9,1)$ & $0,30^{\dagger}$ \\
\hline Insuficiencia renal & $12(14,1)$ & $6(20)$ & $6(10,9)$ & 0,25 \\
\hline Insuficiencia cardiaca & $7(8,2)$ & $2(6,6)$ & $5(9,1)$ & $0,70^{+}$ \\
\hline Cirrosis & $8(9,4)$ & $3(10)$ & $5(9,1)$ & $0,66^{\dagger}$ \\
\hline Anemia & $17(20)$ & $9(30)$ & $8(14,5)$ & 0,09 \\
\hline Malnutrición ${ }^{* *}$ & $13(15,3)$ & $7(23,3)$ & $6(10,9)$ & 0,13 \\
\hline Otros & $46(54,1)$ & $20(66,7)$ & $26(47,3)$ & 0,35 \\
\hline \multicolumn{5}{|l|}{ Malignidad } \\
\hline Tumor sólido & $9(10,6)$ & $3(10)$ & $6(10,9)$ & $0,90^{+}$ \\
\hline Hematológica & $3(3,5)$ & $2(6,6)$ & $1(1,8)$ & $0,26^{\dagger}$ \\
\hline \multicolumn{5}{|l|}{ Complicaciones } \\
\hline Falla respiratoria & $16(18,8)$ & $7(23,3)$ & $9(16,4)$ & 0,43 \\
\hline Shock séptico & $25(29,4)$ & $11(36,7)$ & $14(25,4)$ & 0,28 \\
\hline SDRA & $9(10,6)$ & $4(13,3)$ & $5(9,1)$ & $0,54^{\dagger}$ \\
\hline Injuria renal aguda & $16(18,8)$ & $8(26,6)$ & $8(14,5)$ & 0,17 \\
\hline DHE & $15(17,6)$ & $10(33,3)$ & $5(9,1)$ & $<0,01^{\dagger}$ \\
\hline Trastorno sensoriott & $20(23,5)$ & $10(33,3)$ & $10(18,2)$ & 0,12 \\
\hline Falla hepática & $2(2,4)$ & $1(3,3)$ & $1(1,8)$ & $0,66^{\dagger}$ \\
\hline Otros & $8(9,4)$ & $3(10)$ & $5(9,1)$ & $0,90^{+}$ \\
\hline
\end{tabular}

BLEE: beta lactamasa de espectro extendido; BLEE (+): cepas productoras de BLEE; BLEE (-): cepas no productoras de BLEE.

$\mathrm{VIH}$, virus de inmunodeficiencia humana.

SDRA, síndrome de distrés respiratorio agudo.

DHE: desequilibrio electrolítico.

CVC: catéter venoso central.

* Prueba de chi cuadrado.

+ Prueba exacta de Fisher.

** Índice de masa corporal menor a 18

tt Transtorno del sensorio no presente previamente.

Se encontró una mortalidad total (cruda) de 44,7\% $(38 / 85)$; el $50 \%(19 / 38)$ de estas muertes ocurrieron en el grupo BLEE. Al limitarla a mortalidad atribuible a infección, la mortalidad fue de $41,2 \%$ (35/85), de las cuales el 54,3\%(19/35) ocurrieron en el grupo BLEE.

En el análisis de mortalidad cruda se encontró una mayor proporción de fallecidos por bacteriemia en el grupo de cepas productoras de BLEE en comparación con el grupo de cepas no productoras $(63,3 \%$ [19/30] y $34,5 \%$ [19/55]), lo cual implicó un riesgo relativo (RR) de 1,8 (IC95\%: 1,2-2,9, p=0,009). En el analisis multivariado 
Tabla 3. Factores asociados a la mortalidad en pacientes con bacteriemia por cepas productoras de BLEE.

\begin{tabular}{|c|c|c|c|c|c|c|c|c|c|c|c|c|}
\hline & \multicolumn{6}{|c|}{ Mortalidad Cruda } & \multicolumn{6}{|c|}{ Mortalidad Atribuible } \\
\hline & \multicolumn{3}{|c|}{ Análisis Bivariado } & \multicolumn{3}{|c|}{ Análisis Multivariado } & \multicolumn{3}{|c|}{ Análisis Bivariado } & \multicolumn{3}{|c|}{ Análisis Multivariado } \\
\hline & $\mathbf{R} \mathbf{R}$ & IC95\% & $\mathrm{p}$ & $\mathbf{R} \mathbf{R}$ & IC95\% & $\mathbf{p}$ & $\mathbf{R} \mathbf{R}$ & $\mathrm{IC} 95 \%$ & $\mathrm{p}$ & $\mathbf{R R}$ & IC95\% & $\mathbf{p}$ \\
\hline Producción de BLEE & 1,8 & $1,2-2,9$ & $<0,01$ & 1,5 & $1-2,3$ & 0,04 & 2,2 & $1,3-3,6$ & $<0,01$ & 1,9 & $1,2-2,9$ & $<0,01$ \\
\hline Edad & 1,01 & $1,0-1,02$ & 0,04 & 1,01 & $0,9-1,02$ & 0,61 & 1,01 & $1,00-1,02$ & 0,02 & 1,01 & $0,9-1,02$ & 0,15 \\
\hline Adultos mayores* & 1,7 & $1,0-2,6$ & 0,03 & 1 & $0,5-2$ & 0,90 & 1,7 & $1,1-2,8$ & 0,03 & 0,7 & $0,4-1,4$ & 0,34 \\
\hline Género masculino & 1,4 & $0,9-2,3$ & 0,16 & - & - & - & 1,4 & $0,8-2,4$ & 0,22 & - & - & - \\
\hline Admisión a UCl & 2,1 & $1,4-3,1$ & $<0,01$ & 0,8 & $0,5-1,2$ & 0,30 & 2,1 & $1,3-3,3$ & 0,002 & 0,8 & $0,5-1,2$ & 0,16 \\
\hline Inmunodeficiencia $^{\dagger}$ & 1,2 & $0,7-1,9$ & 0,53 & - & - & - & 1,2 & $0,7-2,0$ & 0,58 & - & - & - \\
\hline $\mathrm{VIH}^{* *}$ & 1,6 & $0,9-2,7$ & 0,09 & - & - & - & 1,4 & $0,7-2,7$ & 0,3 & - & - & - \\
\hline Catéter vesical & 4,4 & $1,9-10,2$ & $<0,01$ & 1,9 & $0,7-5,4$ & 0,20 & 5,2 & $2,0-13,4$ & $<0,01$ & 1,5 & $0,4-4,9$ & 0,53 \\
\hline CVC & 4,1 & $2,3-7,0$ & $<0,01$ & 2,4 & $1,2-4,8$ & 0,01 & 5,6 & $2,9-10,8$ & $<0,01$ & 3,8 & $1,6-8,8$ & $<0,01$ \\
\hline Ventilación mecánica & 2,6 & $1,8-3,6$ & $<0,01$ & 1,6 & $1-2,3$ & 0,03 & 2,5 & $1,7-3,8$ & $<0,01$ & 1,5 & $1-2,3$ & 0,05 \\
\hline Diabetes mellitus & 0,7 & $0,3-1,5$ & 0,32 & - & - & - & 0,5 & $0,2-1,5$ & 0,21 & - & - & - \\
\hline Hipertensión arterial & 0,9 & $0,4-2,0$ & 0,76 & - & - & - & 1 & $0,4-2,2$ & 0,94 & - & - & - \\
\hline Insuficiencia renal & 1,1 & $0,6-2,1$ & 0,68 & - & - & - & 1,3 & $0,7-2,4$ & 0,48 & - & - & - \\
\hline Anemia & 1,1 & $0,6-1,9$ & 0,83 & - & - & - & 1,1 & $0,6-1,9$ & 0,83 & - & - & - \\
\hline
\end{tabular}

$\mathrm{RR}$, riesgo relativo; IC, intervalo de confianza; BLEE, beta lactamasa de espectro extendido; UCI, unidad de cuidados intensivos; $\mathrm{VIH}$, virus de inmunodeficiencia humana; CVC, catéter venoso central.

* 65 años o más

† Presencia de uno o más de: enfermedad autoinmune, corticoterapia, infección por VIH o neutropenia

* Se consideraron a todos los pacientes VIH positivos, independientemente del estadío de la infección

se encontró que el RR ajustado (RRa) de la producción de BLEE para mortalidad cruda fue 1,53 (IC95\%: 1,0-2,3, $\mathrm{p}=0,041)$, el likelihood ratio test (LRt) mostró que el modelo multivariado explica mejor la mortalidad cruda por cepas productoras de BLEE $(p<0,001)$.

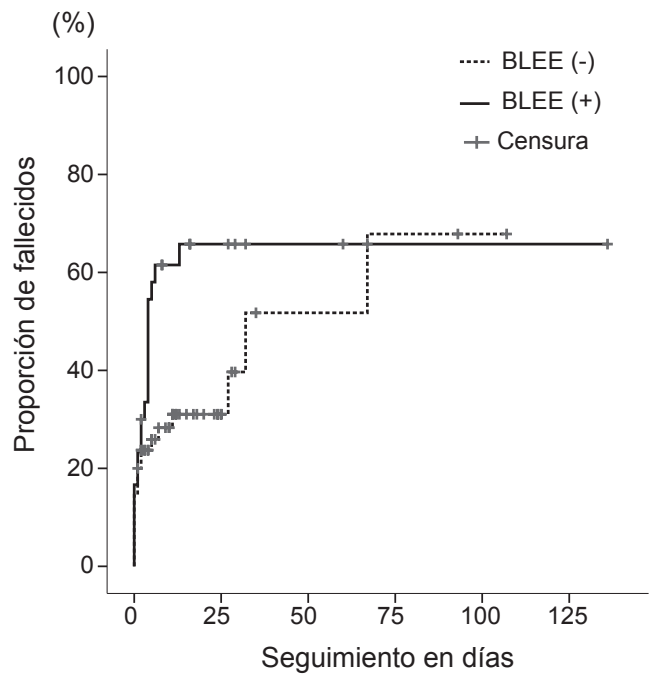

Figura 1. Curva de mortalidad de Kaplan-Meier para pacientes con diagnóstico de bacteriemia por E. coli y Klebsiella spp. estratatificados de acuerdo a la producción o no de BLEE por la cepa aislada.

BLEE: beta lactamasa de espectro extendido. BLEE (+): cepas productoras de BLEE; BLEE (-): cepas no productoras de BLEE. El día 0 corresponde al diagnóstico por hemocultivo, los desenlaces al fallecimiento, y las censuras al alta hospitalaria.
En el análisis de la mortalidad atribuible mostró una mayor proporción de fallecidos en el grupo productor de BLEE en comparación al grupo de cepas no productoras $(63,3 \%[19 / 30]$ y $29,1 \%$ [16/55]), lo cual implicó un $\mathrm{RR}=2,2$ (IC95\%: 1,3-3,6, p=0,002). El RRa de cepas productoras de BLEE para mortalidad atribuible fue de 1,9 (IC95\%: 1,2-2,9, p=0,004), el LRt mostró que el modelo multivariado explica mejor la mortalidad atribuible por cepas productoras de BLEE $(p<0,001)$.

Además de la producción de BLEE, los únicos factores independientes que se asociaron significativamente a mortalidad después de la regresión multivariada fueron el uso de CVC y haber estado en ventilación mecánica. Para el primer caso, el RRa fue 2,4 (IC95\%: $1,2-4,8, p=0,013$ ) para mortalidad cruda y 3,8 (IC95\%: $1,6-8,8, p=0,002)$ para mortalidad atribuible. En el caso de los pacientes que estuvieron en ventilación mecánica, el RRa fue 1,5 (IC95\%: 1,04-2,3, p=0,032) para mortalidad cruda y 1,5 (IC95\%: 1,01-2,3, p=0,047) para mortalidad atribuible.

El analisis de mortalidad se realizó empleando las curvas de Kaplan Meir, el grupo productor de BLEE tuvo una mortalidad inicial mayor. El exceso en mortalidad observado en pacientes con cepas productoras de BLEE se evidenció durante los primeros 12 días de hopitalización pero no persistió a lo largo del seguimiento, encontrándose intersección de las curvas de mortalidad después del día 70 (Figura 1). La intersección de las 
curvas muestra ausencia de riesgos proporcionales entre ambos grupos.

\section{DISCUSIÓN}

Los organismos productores de BLEE son una causa creciente de infecciones adquiridas en la comunidad, no obstante, la mayoría de estudios muestran que la mayor parte de los patógenos productores de BLEE son de origen nosocomial, y aquellos de origen comunitario constituyen una pequeña proporción (24). Un hallazgo interesante en la presente cohorte fue que tanto en la cohortes de cepas productoras de BLEE como en las no productoras, el origen comunitario fue mayor que el nosocomial; por ello, es importante considerar a los organismos productores de BLEE como parte de la sospecha diagnóstica en infecciones severas adquiridas en la comunidad, y tener en cuenta este hecho para planear tratamientos empíricos iniciales, especialmente considerando que BLEE podría ser un factor de mortalidad temprana. Adicionalmente, en este estudio se encontró que la proporción de cepas productoras de BLEE fue mayor a los reportes existentes en América Latina ${ }^{(6,17,24)}$, y la proporción anual se incrementó año a año; la alta tasa de producción de BLEE podría estar en relación a que nuestro estudio solo estudió los casos de bacteriemias, entidad en la cual es más probable encontrar cepas productoras de BLEE, no obstante, la proporción fue elevada incluso en comparación con otros estudios similares.

Consideramos que estos hechos, el incremento de bacteriemias de origen comunitario y la mayor proporción de cepas BLEE, se podrían deber al uso excesivo de cefalosporinas de amplio espectro y la falta de control de uso de antibióticos en nuestra realidad. Existen estudios que respaldan estas suposiciones, Winokur et al. ${ }^{(14)}$ encontraron una mayor prevalencia de BLEE y mayores concentraciones mínimas inhibitorias para cefalosporinas de tercera generación en cepas aisladas en Latinoamérica comparadas con la región del Pacífico Oriental, Europa, Estados Unidos y Canadá. Se ha propuesto que la mortalidad temprana en bacteriemia por cepas BLEE podría explicar este hecho ${ }^{(15)}$. Este planteamiento se aplica a nuestra cohorte, ya que el tiempo entre diagnóstico de bacteriemia y fallecimiento fue más corto en el grupo BLEE y tanto la regresión como las curvas de mortalidad indicaron que BLEE podría ser considerado un factor de mortalidad temprana. La alta tasa de producción de BLEE es un problema importante ya que los antibióticos comúnmente empleados para tratar empíricamente infecciones severas son principalmente cefalosporinas de tercera generación, frente a las cuales estos patógenos estan generando resistencia, y las alternativas terapeuticas adecuadas, como los carbapenémicos, no son fácilmente disponibles en el Perú por su alto costo.

Previamente se ha señalado la heterogeneidad en relación a los resultados de mortalidad asociada a bacteriemias por cepas productoras de BLEE. En nuestro estudio después de ajustar los factores relacionados al paciente y a su hospitalización, el riesgo de mortalidad fue significativamente mayor en la cohorte con producción de BLEE, este hecho se mantiene durante el análisis de la mortalidad atribuible, por lo que nuestro estudio concuerda con aquellos estudios que señalan una mayor mortalidad por estas cepas ${ }^{(17,18,21,22)}$. Además de la producción de BLEE, el antecedente de usar CVC y el haber estado en ventilación mecánica fueron los únicos factores independientemente asociados a mortalidad, y su presencia fue mayor en el grupo productor de BLEE. No obstante, los CVC son a su vez un factor de riesgo para la adquisición de patógenos productores de BLEE ${ }^{(14,20)}$ y su contribución real a la mortalidad debe ser evaluada cuidadosamente.

Los estudios sugieren que la edad (16), admisión a la $\mathrm{UCl}{ }^{(14,19)}$ y otros dispositivos invasivos (4,16) son también factores de riesgo de mortalidad. En nuestro estudio estos factores solo presentaron asociación a mortalidad en el análisis bivariado pero no demostraron significancia después del ajuste multivariado. Además, a pesar de encontrarse mayores porcentajes para el grupo BLEE, la comparación entre las cohortes BLEE y no BLEE no demostró diferencias para factores asociados con la adquisición de cepas BLEE previamente descritos como comorbilidades, inmunosupresión, malignidad y dispositivos invasivos. Aun cuando en nuestro estudio estas diferencias no resultaron ser significativas, estas no deben ser subestimadas ya que la evidencia de su importancia es mayor. Por ello se debe considerar los factores mencionados durante el planteamiento de la sospecha diagnóstica y elección terapéutica.

La tasa total de complicaciones fue mayor en el grupo productor de BLEE, como lo fueron la mayoría de complicaciones individualmente, pero la diferencia no fue significativa. Los pacientes con cepas productoras de BLEE tienden a desarrollar mayores complicaciones intrahospitalarias, lo cual puede relacionarse a la mayor proporción de mortalidad aunque no se encontraron asociaciones individuales significativas. El impacto de las complicaciones en la mortalidad debe ser estudiado junto al tratamiento antibiótico ya que están estrechamente relacionadas. Se deben realizar estudios prospectivos para evaluar la terapia antibiótica ya que es dificil adaptar estudios internacionales a nuestro 
medio debido a las diferencias en el uso de antibióticos en comparación con otras realidades.

La curva de sobrevida de Kaplan-Meier mostró una diferencia grande en mortalidad temprana en favor del grupo de cepas productoras de BLEE, dicha diferencia fue evidente hacia el dia 12 de seguimiento. Sin embargo, las proporciones de mortalidad tendieron a igualarse con el seguimiento. La ausencia de riesgos proporcionales e intersección de las curvas de mortalidad impidió el análisis estadístico de la significancia de la diferencia entre las mismas. No obstante, estos hallazgos apoyan la idea de que la producción de BLEE debe considerarse un factor de riesgo para mortalidad temprana como ha sido propuesto en otros estudios ${ }^{(16)}$, y creemos debe ser considerado por la relevancia del hallazgo.

Dentro de las limitaciones del presente estudio debemos señalar que su naturaleza como diseño de cohorte retrospectiva impide una observación directa y seguimiento del tratamiento antibiótico para su adecuada inclusión en el análisis. Asimismo, algunas diferencias no fueron significativas pese a que la diferencia porcentual fue alta debido, posiblemente, a una baja potencia estadística dado que los eventos eran poco frecuentes, creemos que un estudio más grande discriminaría mejor la tendencia de estas variables. Además, la naturaleza retrospectiva del estudio impide la evaluación de otros factores como la severidad, que no pudo ser evaluada con escalas validadas sino inferida indirectamente a partir de las complicaciones y morbilidades. Tampoco pudo realizarse el análisis de la validez estadística de las curvas de mortalidad al no ser aplicables estas pruebas en curvas con riesgos no proporcionales. Creemos que un incremento en el número de individuos estudiados también discriminaría mejor estas diferencias.
En conclusión, la tasa de producción de BLEE fue más alta en este estudio que en los reportes internacionales, incluso más alta que las tasas descritas en Latinoamérica. La producción de BLEE fue un factor asociado a mortalidad en bacteriemia causada por E. coli y Klebsiella spp, especialmente relacionado a mortalidad temprana atribuible a infección. Los pacientes infectados por cepas BLEE tuvieron no solo mayores tasas de mortalidad sino que desarrollaron más complicaciones. Se puede considerar que una de las principales causas para estos hechos es el uso excesivo de cefalosporinas de amplio espectro y la falta de control de uso de antibióticos en nuestra realidad, por cuanto se debe trabajar en un mejor control de los mismos. Finalmente, se debe tener presente los factores asociados tanto a la producción de cepas productoras de BLEE como a los factores asociados a mortalidad por las mismas durante el planteamiento de la sospecha diagnóstica y la elaboración de la terapéutica inicial en estos casos, con lo cual se podría disminuir la mortalidad por esta causa.

Agradecimientos: a la Dra. Elena Tapia, médico jefe del Servicio de Microbiología del Laboratorio Central del Hospital Nacional Cayetano Heredia, quien permitió la recolección de los datos.

Contribuciones de autoria: todos los autores han participado en la concepción y diseño del artículo así como en la recolección y análisis de los datos. DA participó en la interpretación de los resultados y la redacción del artículo. DA y FS participaron en la revisión crítica del artículo. FS realizó la asesoría estadística. Todos los autores aprueban la versión final a publicar.

Fuentes de financiamiento: autofinanciado.

Conflictos de interés: los autores declaran no tener conflictos de interés.

\section{REFERENCIAS BIBLIOGRÁFICAS}

1. Vallés J, Calbo E, Anoro E, Fontanals D, Xercavins M, Espejo E, et al. Bloodstream infections in adults: importance of healthcare-associated infections. J Infect. 2008;56(1):27-34.

2. Horan TC, Andrus ML, Dudeck MA. CDC/NHSN Surveillance definition of health care-associated infection and criteria for specific types of infections in the acute care setting. Am J Infect Control. 2008;36(5):309-32.

3. Ortega M, Marco F, Soriano A, Almela M, Martínez JA, Muñoz A, et al. Analysis of 4758 Escherichia coli bacteraemia episodes: predictive factors for isolation of an antibiotic-resistant strain and their impact on the outcome. J Antimicrob Chemother. 2009;63(3):568-74.

4. Kang CI, Kim SH, Park WB, Lee KD, Kim HB, Kim EC, et al. Bloodstream infections due to extended-spectrum beta-lactamase-producing Escherichia coli and Klebsiella pneumoniae: risk factors for mortality and treatment outcome, with special emphasis on antimicrobial therapy. Antimicrob Agents Chemother 2004;48(12):4574-81.
5. García P, Rubilar C, Vicentini D, Roman JC, León E, Muñoz G, et al. Caracterización clínica y molecular de bacteriemia $s$ causadas por enterobacterias productoras de betalactamasas de espectro extendido. 2004-2007. Rev Chil Infect. 2011;28(6):563-71.

6. Tumbarello M, Spanu T, Sanguinetti M, Citton R, Montuori E, Leone F, et al. Bloodstream infections caused by extended-spectrum-beta-lactamaseproducing Klebsiella pneumoniae: risk factors, molecular epidemiology, and 
clinical outcome. Antimicrob Agent Chemother. 2006;50(2):498-504.

7. Lautenbach E, Patel JB, Bilker WB, Edelstein PH, Fishman NO. Extended-spectrum beta-lactamaseproducing Escherichia coli and Klebsiella pneumoniae: risk ractors for infection and impact of resistance on outcomes. Clin Infec Dis. 2001;32(8):1162-71.

8. Pitout JD, Laupland KB. Extendedspectrum beta-lactamase-producing Enterobacteriaceae: an emerging public health concern. Lancet Infect Dis. 2008;8(3):159-66.

9. Tumbarello M, Sali M, Trecarichi EM, Leone F, Rossi M, Fiori B, et al. Bloodstream infections caused by extended-spectrum beta-lactamaseproducing Escherichia coli: risk factors for inadequate antimicrobial therapy. Antimicrob Agents Chemother. 2008;52(9):3244-52.

10. Rodríguez-Baño J, Navarro MD, Romero L, Muniain MA, Perea EJ, PérezCano $\mathrm{R}$, et al. Clinical and molecular epidemiology of extended-spectrum beta-lactamase-producing Escherichia coli as a cause of nosocomial infection or colonization: implications for control. Clin Infec Dis. 2006;42(1):37-45.

11. Rodriguez-Baño J, Navarro MD. Extended-spectrum beta-lactamases in ambulatory care: a clinical perspective. Clin Microbiol Infect. 2008;14(Suppl 1):104-10.

12. Mosqueda-Gómez JL, Montaño-Loza A, Rolón AL, Cervantes C, Bobadilladel-Valle JM, Silva-Sánchez J, et al. Molecular epidemiology and risk factors of bloodstream infections caused by extended-spectrum beta-lactamase-producing Klebsiella pneumoniae. a case-control study. Int J Infec Dis. 2008;12(6):653-9.

13. Winokur PL, Canton R, Casellas JM, Legakis N. Variations in the prevalence of strains expressing an extendedspectrum beta-lactamase phenotype and characterization of isolates from Europe, the Americas, and the Western Pacific region. Clin Infect Dis. 2001;32 Suppl 2:S94-103.

14. Szilágyi E, Fuzi M, Borocz K, Kurcz A, Tóth A, Nagy K. Risk factors and outcomes for bloodstream infections with extended-spectrum beta-lactamaseproducing Klebsiella Pneumoniae; findings of the nosocomial surveillance system in hungary. Acta Microbiol Immunol Hung. 2009;56(3):251-62.
15. Du B, Long Y, Liu H, Chen D, Liu $\mathrm{D}, \mathrm{Xu} \mathrm{Y}$, et al. Extended-sprectrumbeta-lactamase-producing Escherichia coli and Klebsiella pneumoniae bloodstream infection: risk factors and clinical outcome. Intensive Care Med. 2002;28(12):1718-23.

16. Memon JI, Rehmani RS, Ahmed MU, Elgendy AM, Nizami IY. Extended spectrum beta-lactamase-producing Escherichia coli and Klebsiella pneumoniae bacteriemia . Risk factors and outcome in the eastern region of Saudi Arabia. Saudi Med J. 2009;30(6):803-8.

17. Ramphal R, Ambrose PG. Extendedspectrum beta-lactamases and clinical outcomes: current data. Clin Infect Dis. 2006;42 Suppl 4:S164-72.

18. Superti SV, Augusti G, Zavascki AP. Risk factors for and mortality of extendedspectrum beta-lactamase-producing Klebsiella pneumoniae and Escherichia coli nosocomial bloodstream infections. Rev Ins Med Trop Sao Paulo. 2009;51(4):211-6.

19. Panhotra BR, Saxena AK, Al-Ghamdi AM. Extended-spectrum beta-lactamaseproducing Klebsiella pneumoniae hospital acquired bacteriemia. Risk factors and clinical outcome. Saudi Med J. 2004;25(12):1871-6.

20. Melzer M, Petersen I. Mortality following bacteraemic infection caused by extended spectrum beta-lactamase (ESBL) producing E.coli compared to non-ESBL producing E. coli. J Infec 2007;55(3):254-9.

21. Bhattacharya S. ESBL-From petri dish to the patient. Indian J Med Microbiol. 2006;24(1):20-4.

22. Cordery RJ, Roberts CH, Cooper SJ, Bellinghan G, Shetty N. Evaluation of risk factors for the acquisition of bloodstream infections with extended-spectrum betalactamase-producing Escherichia coli and Klebsiella pneumoniae species in the intensive care unit; antibiotic management and clinical outcome. J Hosp Infec. 2008;68(2):108-15.

23. Bhavnani SM, Ambrose PG, Craig WA, Dudley MN, Jones RN, SENTRY Antimicrobial Surveillance Program. Outcomes evaluation of patients with ESBL and non-ESBL-producing Escherichia coli and Klebsiella species as defined by CLSI reference methods: report from the SENTRY Antimicrobial Surveillance Program. Diagn Microbiol Infec Dis. 2006;54(3):231-6.
24. Health Protection Agency. UKStandards for Microbiology Investigations. Laboratory detection and reporting of bacteria with extended spectrum betalactamases. London: Health Protection Agency; 2012.

25. Lautenbach E, Schuster MG, Bilker WB, Brennan PJ. The role of chloramphenicol in the treatment of bloodstream infections due to vancomycinresistant Enterococcus. Clin Infec Dis. 1998;27(5):1259-65.

26. Lee SY, Kotapati S, Kuti JL, Nightingale $\mathrm{CH}$, Nicolau DP. Impact of extendedspectrum beta-lactamase-producing Escherichia coli and Klebsiella species on clinical outcomes and hospital costs: a matched cohort study. Infect Control Hosp Epidemiol. 2006;27(11):1226-32.

27. Anthony KB, Fishman NO, Linkin DR, Gasink LB, Edelstein PH, Lautenbacth E. Clinical and microbiological outcomes of serious infections with multidrug-resistant gram negative organisms treated with tigecycline. Clin Infect Dis. 2008;46(4):567-70.

28. Skippen I, Shemko M, Turton J, Kaufmann ME, Palmer C, Shetty N. Epidemiology of infections caused by extended-spectrum beta-lactamaseproducing Escherichia coli and Klebsiella spp.: a nested case-control study from a tertiary hospital in london. J Hosp Infec. 2006;64(2):115-23.

29. Tumbarello M, Sanguinetti M, Montuori E, Trecarichi EM, Posteraro B, Fiori B, et al. Predictors of mortality in patients with bloodstream infections caused by extended-spectrum-beta-lactamase-producing Enterobacteriaceae: importance of inadequate initial antimicrobial treatment. Antimicrob Agents Chemother. 2007;51(6): 1987-94.

30. National Committee for Clinical Laboratory Standards. Performance standards for antimicrobial susceptibility testing. 12th informational supplement. Approved standard M100-S12. Wayne, PA: National Committee for Clinical Laboratory Standards; 2002.

Correspondencia: Diego Adrianzén Herrera

Dirección: Calle El Inkario 238 Int. 301, Derby de Monterrico, Lima 33, Perú.

Teléfono: 975158171

Correoelectrónico:diego.adrianzen@upch.pe 